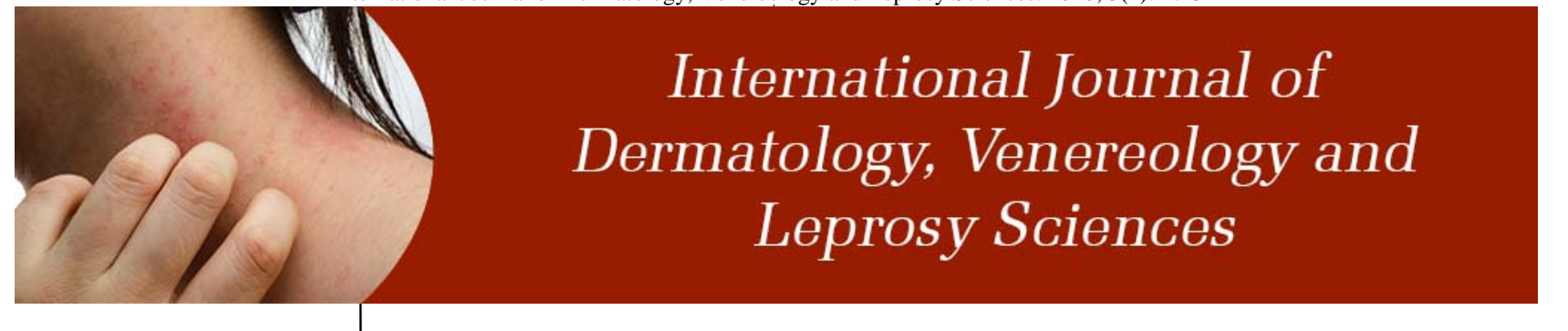

E-ISSN: 2664-942X

P-ISSN: 2664-9411

www.dermatologypaper.com

Derma 2020; 3(1): 27-31

Received: 17-11-2020

Accepted: 20-12-2020

Priscila Regina Orso Rebellato Mackenzie Evangelical University Hospital, Curitiba, Brazil

Deborah Skusa De Torre Mackenzie Evangelical University Hospital, Curitiba, Brazil

Graziela Junges Crescente Rastelli

Mackenzie Evangelical University Hospital, Curitiba, Brazil

Juliano Vilaverde Schmitt UNESP Medical School, Botucatu, Brazil

Cinthia Regina Medaglia Mackenzie Evangelical University Hospital, Curitiba, Brazil
Corresponding Author: Priscila Regina Orso Rebellato Mackenzie Evangelical University Hospital, Curitiba, Brazil

\section{Calcium hydroxylapatite for collagen biostimulation in the neck}

\author{
Priscila Regina Orso Rebellato, Deborah Skusa De Torre, Graziela \\ Junges Crescente Rastelli, Juliano Vilaverde Schmitt and Cinthia Regina \\ Medaglia
}

DOI: $\underline{\text { https://doi.org/10.33545/26649411.2020.v3.i1a.35 }}$

\begin{abstract}
Calcium hydroxylapatite (CaHA) is intended to volumize and to stimulate neocollagenesis, and it is effective to treat facial and extrafacial regions. This study was conducted with 5 patients with an aged cervical region. Photographs and biopsies were performed before treatment and 5 months later. The filler was injected into the subdermal plane of the anterior cervical region. Photos were analyzed by 10 dermatologists and the patient satisfaction was questioned. According to the specialists there was a reduction in aging $(\mathrm{p}=0.001)$, although there was no difference between the patient satisfaction scores $(p=0.576)$. Histology showed that the collagen fibers were thick and irregular in both pre and post treatment, but in most cases, we noticed more compact collagen and elastic fibers afterwards, with new areas of horizontalization. CaHA is effective in improving the appearance and the biostimulation of collagen in the cervical region.
\end{abstract}

Keywords: Calcium hydroxylapatite, neck, skin laxity, neocollagenesis

\section{Introduction}

Imperfections in the neck become a cause of concern by the constant interest in rejuvenation procedures to improve face appearance. This happens because the neck can reveal a person's age as reliably as the face, but it is still neglected in terms of treatments.

Women in their 40s and 50s are more prone to aging in this area as a result of estrogen deficiency and other hormonal changes relating to menopause. These changes lead to an accelerated reduction of collagen and elastin, skin thinning and laxity, worsening lines, folds and wrinkles.

Calcium hydroxylapatite (CaHA) is an effective option of treatment for many areas of facial soft-tissue augmentation and it is associated with a high and well-established safety profile. Treatment provides immediate correction that is gradually followed by new tissue formation through neocollagenesis, dermal cell proliferation, elastin production and angiogenesis. It results in long-lasting aesthetic effect with tight and elastic skin, and increased skin thickness.

The CaHA was first approved in 2006 by the United States Food and Drug Administration (FDA) for the correction of moderate to severe facial wrinkles and folds and for the restoration of the signs of facial fat loss (lipoatrophy) in patients with human immunodeficiency virus ${ }^{[1]}$.

This filler is a synthetic product composed of 25 to $45 \mu \mathrm{m}$ microspheres of calcium hydroxylapatite (30\%) suspended in a gel carrier (70\%) consisting primarily of water, glycerin and carboxymethylcellulose ${ }^{[2,3,4]}$. CaHA is sterile, latex free, synthetic and semipermanent ${ }^{[4]}$. It is composed of minerals that occur naturally in bone and teeth (calcium and phosphate), that is why CaHA is biocompatible ${ }^{[2]}$.

Over time, the carrier gel is gradually absorbed, releasing the CaHA microspheres slowly into the close extracellular matrix with a smoothening effect on the skin and a new collagen production $^{[5]}$. 
CaHA is a filler with high viscosity and mixed with lidocaine increases its malleability, ease of molding, comfort to the patient and decreases the extrusion force. A customized reconstruction of the product increases its versatility and minimizes adverse events.

Acting as a dual-function filler, CaHA is intended to volumize and to stimulate neocollagenesis, what explains why it has many on and off-label applications in clinical practice.

The aim of this study is to investigate the safety, subject satisfaction and efficacy of diluted CaHA in improving skin laxity and the appearance of the neck. The evidence of collagen biostimulation was examined through histopathological study.

\section{Materials and Methods}

This was a single-arm, prospective clinical study performed at a single center in Brazil. Inclusion criteria included subjects older than 40 years and less than 80 years old, without associated severe comorbidities, absence of coagulation disorders or previous history of healing problems. Exclusion criteria consisted of current pregnancy or breastfeeding, severe autoimmune disease associated, smoking, and poor understanding. Written informed consent was obtained from each subject and the study was performed with prior authorization from the Institution and in line with local Research Ethics Committee.

Digital photographs and a skin biopsy in the submental region were performed in each patient before and 5 months after the treatment. All photos were taken with the same camera, in the same conditions of brightness, resolution and distance.

Prior to the procedure, the filler was reconstituted in a 3:1 ratio and homogenized. This dilution was performed with $1.5 \mathrm{ml}$ of CaHA (Radiesse ${ }^{\circledR}$, Merz North America, INC., Raleigh, NC, USA), $0.5 \mathrm{ml}$ of $2 \%$ lidocaine solution without vasoconstrictor and $2.5 \mathrm{ml}$ of $0.9 \%$ saline solution. This reconstituted solution was injected subdermally, with a 70 mm-long 22G cannula, in microdroplets using a fanning technique, in the amount of $1.5 \mathrm{ml}$ on each side of the neck and in the medial region, totalizing $4.5 \mathrm{ml}$ per subject (figure 1).

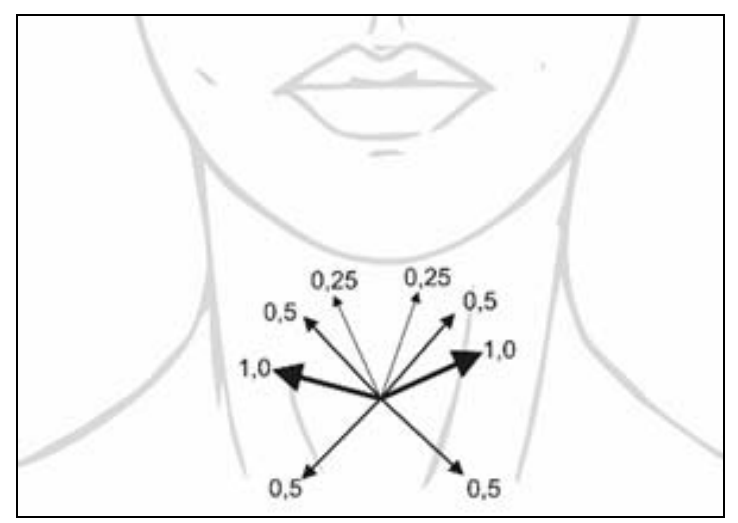

Fig 1: Illustrative diagram of the filler application in the anterior cervical region

A comparison was made between the photos of the preprocedure and 5 months later (figures 2 and 3) for the clinical analysis of the results. These photos were exposed to 10 dermatologists, who evaluated the degree of skin laxity and the wrinkling of each image (absent, mild/moderate, intense, very intense), without knowing when they were taken. Later, the pre and post photos of each patient were paired and re-evaluated by the same dermatologists. At this point, they were questioned: "what is your impression in terms of improvement in the skin sagging and aging of this patient's neck ?. The answers could be none, discreet, significant or very significant.

A comparative study of the biopsies of the pre- and posttreatment was made by a single pathologist. After seven days of material fixating in $10 \%$ formaldehyde, the slides were stained by Hematoxylin-eosin and Weigert methods. Photos were taken by a Sony® camera from a Nikon E200 microscope coupled to an image pickup and analyzed in the scanned image analyzer and Image Proplus ${ }^{\circledR}$ software aiming the histological evaluation of dermal changes produced by CaHA.

The satisfaction of each subject was also analyzed through a standardized questionnaire with questions related to daily activities and clothing (Table 1).

\section{Tab 1. Questionnaire}

1. When going to a social event, how often do you choose clothes or accessories concerned about the appearance of your neck?

2. How often do you use cosmetics, creams and makeup trying to hide the flaccid or aged appearance of your neck?

3. How much do you believe the skin on your neck contributes to give you an older look nowadays?

4. How much sagging do you think your neck skin is?

Continuous variables were analyzed by the parametric $t$ tests of paired and non-parametric Wilcoxon students, depending on the normality of the distributions, as evaluated by the Shapiro-Wilk test. Categorical variables were compared by qui-square or Fisher's exact tests, according to the smallest number of events obtained in each analysis. Correlations were analyzed by the Pearson or Spearman tests, according to the normality of the distributions. Categorical data was represented absolutely. The internal consistency of the questionnaires was evaluated by the Cronbach coefficient. Two-tailed values of $p<0.05$ were considered significant. Data was tabulated by the Microsoft Office Excel 365 program and analyzed by the IBM SPSS 20 program.

\section{Results}

A total of 5 subjects were enrolled in this study. All of them were female, age between 48 and 64 years old, with 2 subjects Fitzpatrick skin Type II, and 3 subjects skin Type III.

In the expert evaluations regarding the analysis of the degree of laxity and wrinkling of the cervical region of each subject, without knowing when each photo was taken, we found that $3 \%$ of the evaluators responded as absent $(100 \%$ of these photos were taken in the post-procedure), $37 \%$ mild/moderate (39\% of these were pre and $61 \%$ postprocedure), $42 \%$ intense (50\% of the pre and $50 \%$ of the post-procedure) and $18 \%$ very intense (78\% of the photos were from before and $22 \%$ from after).

When evaluating the improvement in sagging and in aging of the neck skin in the paired photos (pre and post), $10 \%$ of the evaluators answered none, 34\% discrete, 38\% significant and $18 \%$ very significant (figure 2 and 3 ). 

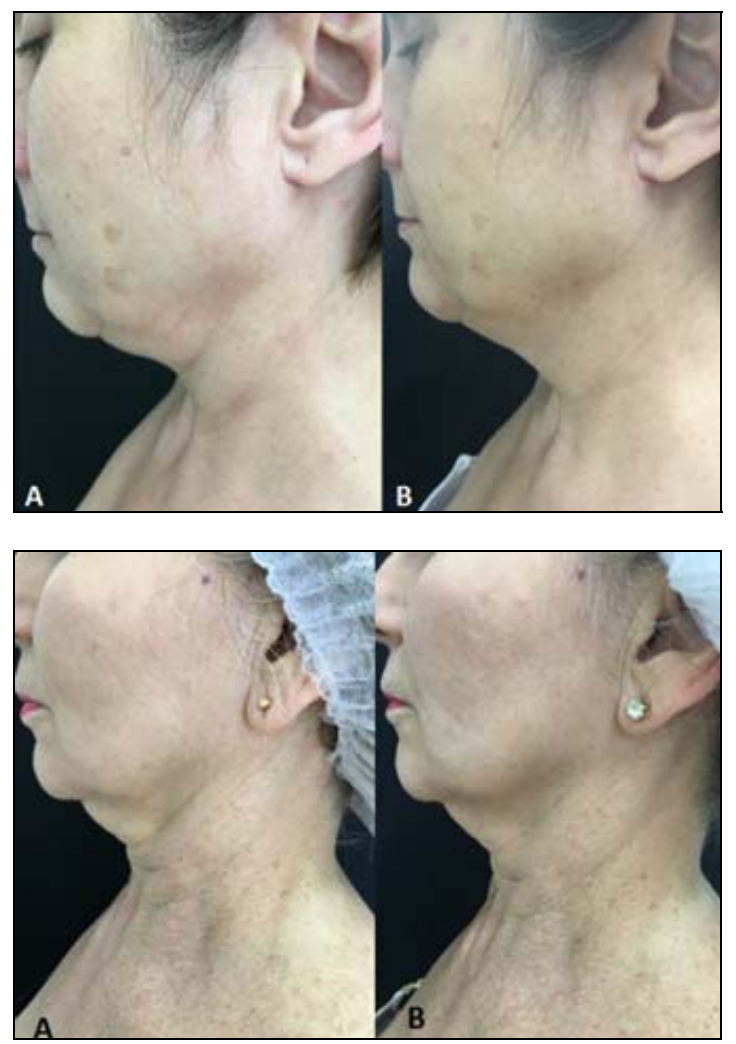

Fig 2 and 3: Before (A) and after 5 months (B) photos of a single application of calcium hydroxylapatite in the cervical region
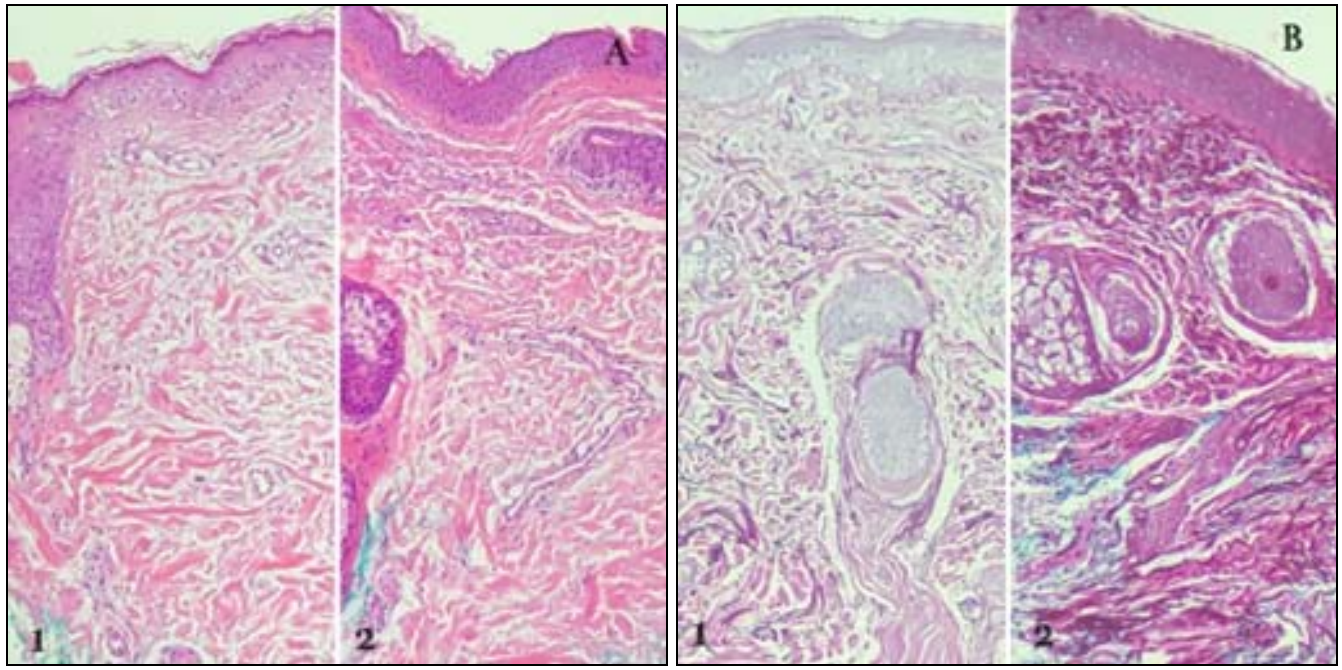

Fig 4 and 5: Comparative photos from before (1) and after treatment (2) in hematoxylin-eosin (A) and Weigert (B).10x

Mild pain was experienced by $60 \%$ of subjects during the procedure, and only one participant experienced brushing, which resolved in 7 days. No other adverse events were reported.

\section{Discussion}

The results of this clinical study confirm that diluted CaHA improves skin laxity and promotes collagen biostimulation. For more than a decade, CaHA has been used for HIVassociated lipoatrophy and facial rejuvenation, but recent studies have shown that when injected more superficially in its diluted or hyper-diluted form, it is also effective in treating volume loss and skin laxity in non-facial areas.
In general, dermatologists evaluated a reduction in the degree of aging ( $p=0.001$ ), according to a generalized linear mixed model with gamma distribution, controlled by patients and evaluators (Akaike corrected: 71 427). And the intraclass correlation among the evaluators (mean measures -absolute agreement) was 0.861 (satisfactory).

According to the data obtained, it can be affirmed that there was no statistically significant difference between the degree of satisfaction of the participants with the result, when compared before and after the procedure $(p=0.576)$. When questioned about the influence of their cervical region in daily activities and clothing, 3 patients showed reduced dissatisfaction with the appearance of the cervical region, and 2 showed an increase (Cronbach's alpha of 0.74).

The epidermis did not present significant alterations, showing no change in its thickness or in the epidermal cones. No significant difference was observed in the dermal vascularization and in the presence of inflammatory cells in the perivascular space.

The collagen fibers presented thickened and irregular in all cases (before and after). However, in most cases (4 cases), more compact collagen fibers were noticed in the postprocedure (decrease in the spacing between them).

The elastic fibers (shown in Weigert's stain) were thickened and irregular, with fragmentation (both before and after). However, in 2 of the patients, the elastic fibers increased in quantity after the treatment, with areas of horizontalization previously unobserved (Figure 4 and 5). 
aging of the neck skin, being that $90 \%$ of the evaluators found some degree of such improvement in the analyzed photos, even after only one session. A Russian study with 20 subjects concluded that diluted CaHA is very effective for skin tightening of the neck and décolletage. It demonstrated an increase in skin elasticity, by cutometry, and improvement in dermal thickness, with ultrasound ${ }^{[7]}$. Another publication showed, after only a single treatment, that diluted $\mathrm{CaAH}$ is an effective procedure for skin tightening in the upper arms and abdomen ${ }^{[8]}$.

Although the present results have not shown difference between satisfaction degree of the participants, when compared before and after the procedure, 3 patients reduced dissatisfaction with the appearance of the cervical region during daily activities and clothing. This ability of CaHA to produce satisfactory and visible aesthetic improvements in the skin, by dermal remodeling, has also been demonstrated in other recent studies ${ }^{[7,8,9,10]}$. They evaluated the results of using diluted CaHA in upper arms, abdomen, neck, décolletage, buttocks and thighs.

A recent study showed, through morphometric analysis, that following CaHA treatment, the proportion of thin newly formed collagen type III increased $(p<0,01)$ in comparison to the proportion of thick mature collagen type I fibers. ${ }^{11}$ Another publication demonstrated a stimulatory effect on fibroblasts, the constitutive resident cells of the connective tissue, responsible for the formation and turnover of the extracellular matrix components, after two months from CaHA injection. They observed that these new fibroblasts are rich in rough endoplasmic reticulum profiles, what represents the molecular precursors (procollagen) of fibrillary components of the extracellular matrix (mainly collagen). The results of this study also demonstrated that the specific modifications of the CaHA filler injected are due to extracellular interactions of big cells surrounding the filler microspherules with their constitutive material, supporting a mechanism without phagocytosis of the injected material, and a slowly released filling the extracellular matrix, with a long smooth action ${ }^{[5]}$.

Elastin is another structural component of the dermis, providing its elasticity and flexibility. A Russian pilot study found that the CaHA injection is followed by increased elastin expression in parallel with collagen type I. There was also increased expression of CD34, a marker of angiogenesis, suggesting that the dermal remodeling was accompanied by outgrowth of new blood vessels, vital to supply nourishment to newly forming tissues during remodeling and repair ${ }^{[7]}$.

The present observations showed no epidermis significant alterations and no significant difference in the dermal vascularization. The collagen fibers presented thickened and irregular in all cases. However, in most cases, there were more compact collagen fibers in the post-procedure, with a reduced space between them. The elastic fibers were fragmented in both before and after evaluations, but they increased in quantity after the treatment in 2 patients, with new areas of horizontalization.

The evaluation of the improvement of skin density and thickness in our study was subjective by clinical photos. Specialists analyzed our results through their impression of the sagging and aging of the skin of patient's neck. $90 \%$ found some degree of improvement in comparison to pre and post-treatment. When experts observed the degree of laxity and wrinkling of the cervical region of each participant, most of the photos with improvement in appearance were the ones taken in the post-procedure.

A study which investigated the efficacy of CaHA in correcting skin flaccidity in the thighs, abdomen and brachial zones showed improvements in $78 \%$ of cases five weeks after treatment through ultrascan measurements. Abdomen showed the best response ${ }^{[12]}$. Other publication evidenced by cutometry results for upper arm skin an increase in elasticity from $72 \mathrm{U}$ to $82 \mathrm{U}(p<0,05)$ after three months of injection of diluted CaHA and, by ultrasound measures of the abdominal wall an augment in dermal thickness of $26,7 \%(p<0,05)^{[8]}$.

This treatment was well tolerated, with no cases of severe adverse events. They were limited to mild pain and transient brushing, similar to adverse events observed in other skin tightening studies ${ }^{[4,7,9,10,12]}$.

The long-term maintenance of the esthetic results was not explored in this study, but the longevity of CaHA as a dermal filler in volume restoration has previously been demonstrated. Studies support a corrective effect lasting up to 12 months in the jawline and dorsal hand volume loss [2, 13].

\section{Conclusions}

The results of the present study in investigating the use of diluted CaHA for improving skin laxity and the appearance of the neck were very positive. Although our data were obtained through the first 5 months, the longevity of the correction is estimated to be similar to that of CaHA in other applications. Prospective studies with longer follow up should explore the sustained efficacy of the treatment. The small number of subjects and the subjective nature of the results were also limitations of this study. Future researches can help to answer how many procedures are required for optimal results and if there is or not an ideal patient for this kind of treatment.

\section{References}

1. Goldman MP, Moradi A, Gold MH et al. Calcium hydroxylapatite dermal filler for treatment of dorsal hand volume loss: results from a 12-month, multicenter, randomized, blinded trial. Dermatol Surg. 2018; 44:7583.

2. Faad JE, Faad HS. Aesthetic applications of calcium hydroxylapatite volumizing filler: an evidence-based review and discussion of current concepts: part 1 of 2 . J Drugs Dermatol. 2013; 12(12):1345-1354.

3. Dallara JM, Baspeyras M, Bui P, Catier H, Charavel $\mathrm{MH}$, Dumas L. Calcium hydroxylapatite for jawline rejuvenation: consensus recommendations. J Cosmet Dermatol. 2014; 13(1):3-14.

4. Chao YY, Chiu HH, Howel DJ. A novel technique for horizontal neck lines correction using calcium hydroxylapatite. Dermatol Surg. 2011; 37:1542-1545.

5. Zerbinati N, D' Este E, Parodi PC, Calligaro A. Microscopic and ultraestructural evidences in human skin following calcium hydroxylapatite filler treatment. Arch Dermatol Res. 2017; 309:389-396.

6. Eviatar J, Lo C, Kirszrot J. Radiesse: Advanced tachniques and applications for a unique and versatile implant. Plast Reconst Sug. 2015; 136(5):164-170.

7. Yutskovskaya YA, Kogan EA. Improved neocollagenesis and skin mechanical properties after injection of diluted calcium hydroxylapatite in the neck 
and décolletage: a pilot study. J Drugs Dermatol. 2017; 16(1):68-74.

8. Lapatina NG, Pavlenko T. Diluted calcium hydroxylapatite for skin tightening of the upper arms and abdomen. J Drugs Dermatol. 2017; 16(9):900-906.

9. Casabona G, Pereira G. Microfocused ultrasound with visualization and calcium hydroxylapatite for improving skin laxity and cellulite appearance. Plast Reconstr Surg Glob Open. 2017; 5(7):e1388.

10. Casabona G, Nogueira Teixeira D. Microfocused ultrasound in combination with diluted calcium hydroxylapatite for improving skin laxity and the appearance of lines in the neck and décolletage. $\mathrm{J}$ Cosmet Dermatol. 2018; 17(1):66-72.

11. Zerbinati N, Calligaro A. Calcium hydroxylapatite treatment of human skin: evidence of collagen turnover through picrosirius red staining and circularly polarized microscopy. Clin Cosmet Investing Dermatol. 2018; 15(11):29-35.

12. Wasylkowski VC. Body vectoring technique with Radiesse for tightening of the abdomen, thighs, and brachial zone. Clin Cosmet Investing Dermatol. 2015; 8:267-273.

13. Juhász MLW, Marmur ES. Examining the Efficacy of Calcium Hydroxylapatite Filler with Integral Lidocaine in Correcting Volume Loss of the Jawline-A Pilot Study. Dermatol Surg. 2018; 44(8):1084-1093. 